\title{
Successful Interventional Recanalization against Heavily Calcified Chronic Total Occlusion of Proximal Right Coronary Artery Utilizing Retrograde Guidewire Trapping Technique at the Terminal Aorta
}

\section{Masaki Tanabe*, Takeo Kunitomo and Osamu Doi}

Department of Cardiology, Dai-ni Okamoto General Hospital, Kyoto, Japan

*Corresponding author: Masaki Tanabe, Department of Cardiology, Dai-ni Okamoto General Hospital, Kyoto, Japan, Tel: +81(774)44-4511; Fax: +81(774)46-7822; Email: shaishaibeam@yahoo.co.jp

Rec date: January 08, 2016; Acc date: February 04, 2016; Pub date: February 10, 2016

Copyright: $\odot 2016$ Tanabe M, et al. This is an open-access article distributed under the terms of the Creative Commons Attribution License, which permits unrestricted use, distribution, and reproduction in any medium, provided the original author and source are credited.

\begin{abstract}
We described a case of chronic total occlusion (CTO) of the ostial right coronary artery (RCA) in a 79-year-old woman that had been undergoing hemodialysis.

Coronary intervention of the ostial RCA-CTO was performed via the retrograde approach. The retrograde hard wire was able to pass through the СTO segment via a transseptal collateral and advanced at the ascending aorta. However, the microcatheter tip could not advance into the heavily calcified plaque of the CTO segment. Moreover, it was very difficult to catch the retrograde hard wire tip in the ascending aorta using a $10 \mathrm{~mm}$ Gooseneck snare at the ostium of the antegrade guiding catheter due to three-dimensional size mismatch. Thereby, we attempted to cross the occluded segment directly using a long-shaft soft guidewire designed for retrograde CTO intervention (0.009 inch $330 \mathrm{~cm}$ ). The unassisted long-shaft wire tip was passed successfully through the CTO segment retrogradely. After the retrograde wire tip was pushed ahead of the terminal aorta, it was caught using the former snaring system and another guiding catheter for the antegrade approach was pulled in. After wire externalization was completed, balloon dilatation and stent deployment through the CTO segment were performed successfully via the antegrade approach.
\end{abstract}

Here, we report a case of successful interventional revascularization of a heavily calcified and device-uncrossable CTO lesion by the retrograde wire trapping technique using a gooseneck snare device at the terminal aorta. The method in which the capture of a retrograde wire using Gooseneck snare system may be not novel in itself. But, in case of difficulty in uncrossability devices and catching it at the ascending aorta as well, this combinative technique is beneficial to the ease of wire externalization.

Keywords Percutaneous coronary intervention; Chronic total occlusion; Uncrossable devices; Retrograde guidewire trapping technique

\section{Introduction}

Percutaneous coronary intervention (PCI) of chronic total occlusion (CTO) is still challenging regardless of the expertise of the operator and despite the development of novel interventional devices.

The inability to pass guidewires through CTO lesions is the most common reason for failure of CTO-PCI [1]. However, "deviceuncrossable" CTO lesions even after successful passage of the guidewire account for $6.4 \%-15 \%$ of all CTO-PCI procedural failures [1-3].

Various antegrade techniques have been developed for approaching device-uncrossable CTO lesions [4-7]. On the other hand, the retrograde approach $[8,9]$, which has already become an essential alternative to the classical antegrade method, is also considered an effective technique to overcome problems, such as device-uncrossable CTO lesions.

In this case, we succeeded interventional recanalization of the proximal right coronary artery with a heavily calcified CTO lesion utilizing a retrograde guidewire trapping technique with snare devices at the terminal aorta. This method is helpful for overcoming the difficulty in constructing a retrograde system as well as the problem of device-uncrossable CTO lesions.

\section{Case}

A 79-year-old female patient that had been undergoing hemodialysis for several years due to end-stage renal failure suddenly fell into ventricular fibrillation, and cardiopulmonary resuscitation was performed. After resuscitation, emergent coronary angiography revealed severe stenosis of the proximal left circumflex artery (LCX) with three-vessel disease involving the proximal RCA-CTO. After emergent PCI to the proximal LCX using a drug-eluting stent (DES), the intense discussions regarding her further treatment were performed among the heart team of our institution whether undergo CABG or coronary intervention, because it was mandatory to complete revascularization that the existence of prolonged ventricular tachycardia indicated residual ischemia.

Considering her clinical course, her general status ( Logistic Euro score was $27.93 \%$ ) including some comorbidities having prior cerebral infarction with bilateral carotid artery stenosis, severe impairment of mobility secondary to musculoskeletal, and moderate reduced LV 
Citation: Tanabe M, Kunitomo T, Doi O (2016) Successful Interventional Recanalization against Heavily Calcified Chronic Total Occlusion of Proximal Right Coronary Artery Utilizing Retrograde Guidewire Trapping Technique at the Terminal Aorta. J Cardiovasc Dis Diagn 4: 231. doi:10.4172/2329-9517.1000233

Page 2 of 4

function (LVEF 40\%) and invasion related CABG, interventional revascularization was designed preferentially one week after emergent PCI.

Coronary angiography showed that the RCA had a CTO at the proximal portion and had diffuse stenosis from the aortic ostium with heavy calcification. The left main trunk (LMT) had moderate stenosis and the LCX had residual stenosis at the bifurcation of the LMT. Fair transseptal collaterals were found in the RCA, and it was dyed with contrast media retrogradely up to the mid-portion (Figure 1).

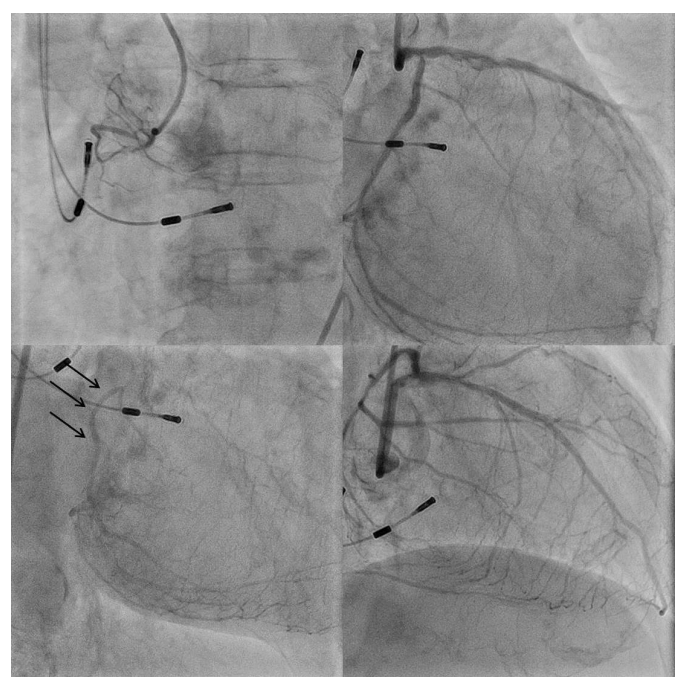

Figure 1: Baseline coronary angiograms. The RCA had CTO at the proximal portion and diffuse stenosis from the aortic ostium with heavy calcification. The LMT had moderate stenosis and LCX had residual stenosis at the bifurcation of the LMT. The RCA was dyed with contrast media up to the mid-portion retrogradely (black arrows). Fair transseptal collaterals from the LCA to thedistal RCA.

Considering that it was mandatory to perform complete revascularization as well as to prevent myocardial damage due to ischemia during procedure, staged PCIs were scheduled to the proximal RCA-CTO, following the LMT bifurcation including the ostial LCX.

The PCI to the proximal RCA-CTO started with insertion via the bifemoral arteries. First, this was attempted via the antegrade approach, but a 7Fr AL1 guide catheter with side holes (Axess; Asahi Intecc Co. Ltd., Aichi, Japan) was unable to stably engage because of severe stenosis with heavy calcification from the aortic ostium. Moreover, it was difficult to maintain the coaxial position of the guide catheter due to anterior take-off. The procedure was changed to the retrograde approach. A 7Fr SPB 3.5 guide catheter with side holes (Axess; Asahi Intecc) was inserted into the ostium of the LMT via the right transfemoral approach. Retrograde wiring by the SUOH (Asahi Intecc) with a Corsair microcatheter (Asahi Intecc) was successful in negotiating from the 3rd septal branch using the wire surfing procedure, and reached the distal true lumen of the RCA. After the Corsair microcatheter progressed retrogradely to the CTO exit, the retrograde wire was exchanged to the Ultimate Bros.3 (Asahi Intecc) for penetration into the CTO segment. The retrograde Ultimate Bros. 3 was successfully passed through all CTO segments and reached the ascending aorta directly. However, the retrograde Corsair microcatheter was could not cross due to the heavily calcified plaque within the CTO segment (Figure 2A). Consequently, it was very difficult to advance the retrograde Ultimate Bros. 3 into the antegrade guide catheter, which showed shallow engagement of the RCA ostium without coaxial support. Moreover, it was also difficult to catch the retrograde hard wire in the ascending aorta using a $10 \mathrm{~mm}$ Gooseneck snare at the ostium of the antegrade guiding catheter due to threedimensional size mismatch despite of trials again and again.

As a bailout procedure, the retrograde wire was changed to a 0.009 " $330 \mathrm{~cm} \mathrm{RG3}$ wire (Asahi Intecc) designed to perform wire externalization retrogradely in CTO intervention. The RG3 was retrogradely passed successfully through the CTO segment, and reached the ascending aorta directly (Figure 2B). However, the wire tip could not be inserted into the antegrade guide catheter. Therefore, the RG3 wire tip was advanced to the terminal aorta in its present condition, and was easily caught by the $10 \mathrm{~mm}$ Amplatz GooseNeck snare system (Covidien Corp., Plymouth, MN) and brought into the antegrade guide catheter at this point (Figure 3 ). The antegrade guide catheter was then reinserted to the RCA ostium with navigation by the RG3 shaft, and wire externalization was completed.

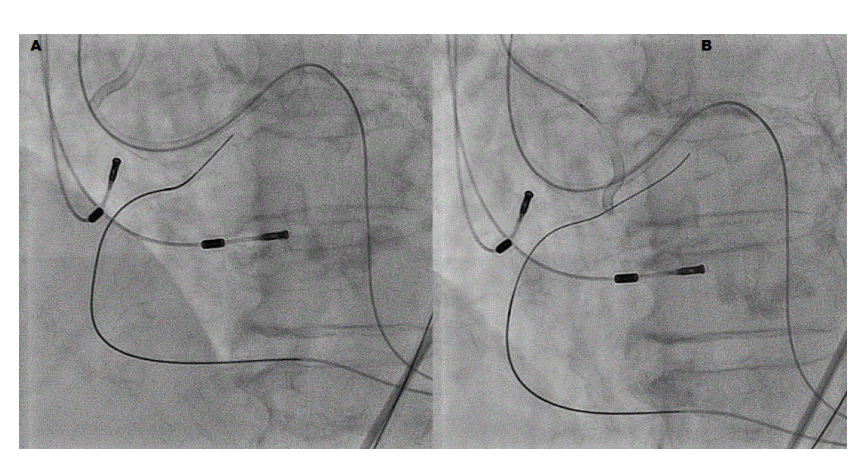

Figure 2: Coronary angiograms during the PCI procedure. The retrograde Ultimate Bros.3 was able to pass through all CTO segments and reached the ascending aorta directly. A: The Corsair microcatheter could not cross due to the heavily calcified plaque within the CTO segment. As a bailout procedure, the retrograde wire was changed to the RG3 wire $(0.009$ inch, $330 \mathrm{~cm}$; Asahi Intecc). B: Consequently, the RG3 was retrogradely successful in passing through the CTO segment again, and approached the ascending aorta directly. However, the wire tip was unable to be inserted into the antegrade guide catheter.

PCI to RCA-CTO was then performed continuously by then antegrade approach. As strong backup force was provided by completing wire externalization, the dedicated low-profile balloon (Ikazuchi X hyper $1.5 \times 9 \mathrm{~mm}$; Kaneka Medix Corp., Osaka, Japan) was successfully crossed completely through the CTO lesion beyond the heavily calcified segment. Although low-profile compliant balloons were easily ruptured due to heavily calcified plaque during dilatation, non-compliant balloons were eventually successfully dilated by high atmospheric pressure without indentation. Subsequently, two Biolimus Eluting Stents (Nobori $3.0 \times 14 \mathrm{~mm}$ and Nobori $3.0 \times 8 \mathrm{~mm}$; Terumo Corp., Tokyo, Japan) were deployed at the CTO lesion (Figure 4A). Finally, successful revascularization was provided to the proximal RCA-CTO lesion (Figure 4B). Summarized information in this CTOPCI was shown as follows; total fluoroscopic time was 254 minutes, total radiation dose was 716 Gray-cm2, and total contrast (Iohexol 350 
Citation: Tanabe M, Kunitomo T, Doi O (2016) Successful Interventional Recanalization against Heavily Calcified Chronic Total Occlusion of Proximal Right Coronary Artery Utilizing Retrograde Guidewire Trapping Technique at the Terminal Aorta. J Cardiovasc Dis Diagn 4: 231. doi:10.4172/2329-9517.1000233

Page 3 of 4

$\mathrm{mgI} / \mathrm{ml}$ ) volume was $305 \mathrm{ml}$. After that, she undertook overnight continuous hemodiafiltration in intensive care unit without complication. PCI to the LMT bifurcation including the ostial LCX was performed using DES implantation one week later and she leaved our hospital without MACCE.

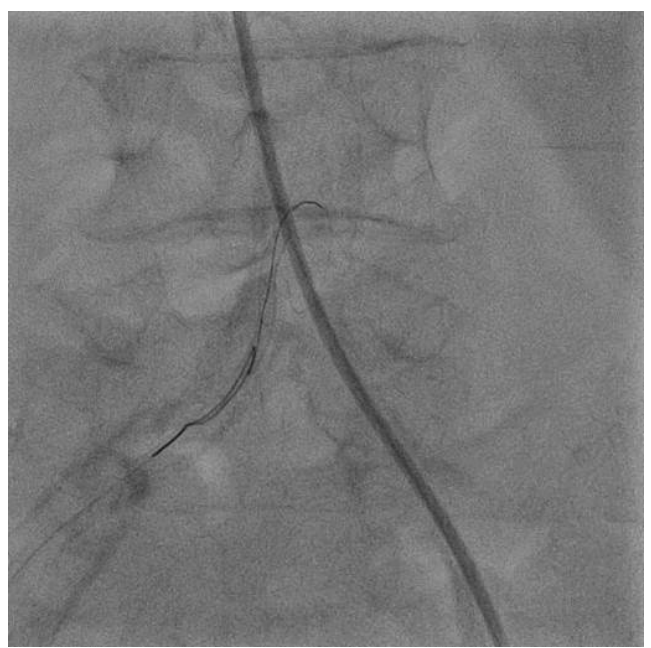

Figure 3: Retrograde guidewire trapping technique at the terminal aorta. The tip of the RG3 wire was pushed forward to the terminal aorta in its present condition, and was caught by the $10 \mathrm{~mm}$ Amplatz GooseNeck snare system (Covidien Corp.) and brought into the antegrade guide catheter at this point.

\section{Discussion}

Here, we described a case of successful interventional revascularization of a heavily calcified RCA with difficulty of passing devices through the CTO lesion utilizing the wire trapping technique with snare devices at the terminal aorta. The method was beneficial for constructing a retrograde system as well as overcoming difficult CTO lesions, e.g., the inability to move devices across the lesion.

Recent studies and meta-analyses have shown that there is significant clinical benefit with improved long-term outcomes in patients undergoing successful CTO revascularization compared to unsuccessful CTO recanalization [10,11]. However, successful interventional recanalization of CTO lesions can often be very challenging. The inability to pass guidewires through CTO lesions is the main reason for failure of CTO-PCI [1]

On the other hand, device-uncrossable CTO lesions, even after successful passage of the wire through the lesion, account for $6.4 \%-15 \%$ of all CTO-PCI procedural failures [1-3] and are most often due to heavy calcification at the occlusion site.

Various techniques via the antegrade approach have been illustrated for approaching device-uncrossable CTO lesions, which can be grouped into two categories: (1) techniques for lesion modification, such as drilling using Tornus catheters [4,5], buddy (occasionally triple) wire technique, cracking technique utilizing stiff wires, excimer laser coronary angioplasty, and rotational atherectomy [2,5]; and (2) techniques that increase guide catheter support, such as child-inmother techniques and various anchor techniques [6]. Occasionally combinations of these techniques are successfully utilized [7].

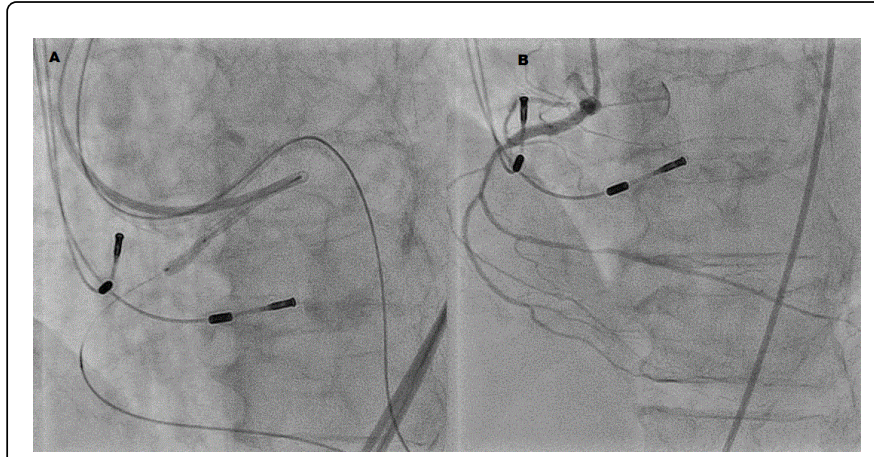

Figure 4: PCI procedures after wire externalization. A: A stent deployed at the CTO segment. B: The final angiogram showed optimal results with restoration of flow in the RCA.

Retrograde CTO recanalization has recently become an essential alternative technique to the classical antegrade approach [8]. The retrograde technique has a high procedural success rate with a low complication profile, despite frequent utilization in the most anatomically and clinically complex cases $[9,12]$. Accordingly, the retrograde approach is also considered to be an effective alternative method for use in device-uncrossable CTO lesions. In cases in which wire externalization has been completed successfully, strong backup support may be provided by the bilateral guide catheter anchor. In a retrograde manner, either pushing the retrograde guidewire into the guiding catheter or trapping it at the contralateral sheet may be used for wire externalization after passing through CTO segments.

In this case, antegrade guide catheters were unable to engage stably due to severe stenosis with heavy calcification from the RCA ostium and anterior takeoff.

In addition, as the CTO was short and the heavily calcified CTO segment was located near the ostium of the RCA, there was very limited space to maneuver antegrade wires if the antegrade guide catheters had been successfully engaged coaxially.

Eventually, in this case, we found a way to capture the retrograde long guidewire from the terminal aorta using $10 \mathrm{~mm}$ gooseneck snare devices and established strong backup support for interventional revascularization of the heavily calcified CTO lesion through which devices could not pass.

The RG3 has a 0.009 " tip and shaft, $330 \mathrm{~cm}$ length. It is not used for penetration, but dedicated for procedural step of wire externalization in the setting of retrograde CTO-PCI. Certainly, the penetration force of the Ultimate Bros. 3 is much stronger than the RG3. Although control of the tip of the RG3 wire was not so good, it is theoretically feasible to manage to slip through the lumen which was successfully penetrated by the tip of the Ultimate Bros.3. In this case, the following procedures were attempted as a bailout, and finally, the RG3 managed to pass successfully through the lumen retrogradely after the Ultimate Bros. 3 passing through the CTO segment.

The retrograde wire trapping technique using snare devices has already been reported in a number of cases with lesions in the ascending aorta $[1,13,14]$. However, in the retrograde trapping technique at the ascending aorta, it was occasionally difficult to catch the guidewire and the procedure was time-consuming due to size mismatch and three-dimensional mismatch. 
Citation: Tanabe M, Kunitomo T, Doi O (2016) Successful Interventional Recanalization against Heavily Calcified Chronic Total Occlusion of Proximal Right Coronary Artery Utilizing Retrograde Guidewire Trapping Technique at the Terminal Aorta. J Cardiovasc Dis Diagn 4: 231. doi:10.4172/2329-9517.1000233

Page 4 of 4

Yokoi et al. [15] reported a novel homemade snare device to adjust the size of the snare guidewires, but it may interfere with the patient's heartbeat. At the terminal aorta, it was easy to anchor with snaring devices, because the retrograde wire tip was not affected by the patient's heartbeat. The retrograde wire trapping technique in the terminal aorta solves the problems associated with interference with heartbeat as well as the time-consuming nature of the procedure.

To our knowledge, this is the first reported of the utilization of a retrograde guidewire trapping technique with snaring devices at the terminal aorta in bilateral CTO-PCI.

In conclusion, we were successful in interventional revascularization of a heavily calcified proximal RCA-CTO segment with deviceuncrossable CTO lesions utilizing the retrograde wire trapping technique with gooseneck snare devices at the terminal aorta. This method was beneficial to construct a retrograde system as well as to overcome difficult device-uncrossable CTO lesions. Moreover, it may solve the problems regarding the long time required for the procured associated with interference with the heartbeat and three-dimensional size mismatch.

\section{References}

1. Stone GW, Colombo A, Teirstein PS, Moses JW, Leon MB, et al. (2005 Percutaneous recanalization of occluded coronary arteries: procedural techniques, devices, and results. Catheter Cardiovasc Interv 66: 217-236.

2. Pagnotta P, Briguori C, Mango R, Visconti G, Focaccio A, et al. (2010) Rotational atherectomy in resistant chronic total occlusions. Catheter Cardiovasc Interv 76: 366-371.

3. Patel SM, Pokala NR, Menon RV, Kotsia AP, Raja V, et al. (2015) Prevalence and treatment of "balloon-uncrossable" coronary chronic total occlusions. J Invasive Cardiol 27: 78-84.

4. Brilakis ES, Banerjee S (2011) Crossing the "balloon uncrossable" chronic total occlusion: Tornus to the rescue. Catheter Cardiovasc Interv 78: 363-365.
5. Pagnotta P, Briguori C, Ferrante G, Visconti G, Focaccio A, et al. (2013) Tornus catheter and rotational atherectomy in resistant chronic total occlusions. Int J Cardiol 167: 2653-2656.

6. Kovacic JC, Sharma AB, Roy S, Li JR, Narayan R, et al. (2013) GuideLiner mother-and-child guide catheter extension: a simple adjunctive tool in PCI for balloon uncrossable chronic total occlusions. J Interv Cardiol 26: 343-350.

7. Kirtane AJ, Stone GW (2007) The Anchor-Tornus technique: a novel approach to "uncrossable" chronic total occlusions. Catheter Cardiovasc Interv 70: 554-557.

8. Surmely JF, Tsuchikane E, Katoh O, Nishida Y, Nakayama M, et al. (2006) New concept for CTO recanalization using controlled antegrade and retrograde subintimal tracking: the CART technique. J Invasive Cardiol 18: 334-338.

9. Joyal D, Thompson CA, Grantham JA, Buller CE, Rinfret S (2012) The retrograde technique for recanalization of chronic total occlusions: a stepby-step approach. JACC Cardiovasc Interv 5: 1-11.

10. Godino C, Bassanelli G, Economou FI, Takagi K, Ancona M, et al. (2013) Predictors of cardiac death in patients with coronary chronic total occlusion not revascularized by PCI. Int J Cardiol 168: 1402-1409.

11. Joyal D, Afilalo J, Rinfret S (2010) Effectiveness of recanalization of chronic total occlusions: a systematic review and meta-analysis. Am Heart J 160: 179-187.

12. Mibiki Y, Kikuta H, Sumiyoshi T, Shibata M, Osawa N (2013) Percutaneous coronary intervention by retrograde approach for chronic total occlusion of the proximal left anterior descending artery via a gastroepiploic artery graft. Cardiovasc Interv Ther 28: 91-97.

13. Otsuka Y, Kataoka Y, Fukuda T (2009) Snaring the retrograde wire in the aortic root for chronic total occlusion after Bentall operation in a patient with Behcet's disease. J Invasive Cardiol 21: E137-140.

14. Ge J, Zhang F (2009) Retrograde recanalisation of chronic total coronary artery occlusion using a novel "reverse wire trapping" technique. Catheter Cardiovasc Interv74: 855-860.

15. Yokoi K, Sumitsuji S, Kaneda H, Siegrist PT, Okayama K, et al. (2015) A novel homemade snare, safe, economical and size-adjustable. EuroIntervention 10: 1307-1310. 